\title{
Política del tratamiento de la información documentaria en bibliotecas universitarias: estudio diagnóstico del contexto en la perspectiva del catalogador y del usuario
}

Policy of documentary information treatment in University Libraries:

diagnostic study of the context in the cataloguer's and user's perspective

\author{
Milena Polsinelli Rubi (1), Mariângela Spotti Lopes Fujita (2), \\ Vera Regina Casari Boccato (3) y Maria Carolina Gonçalves (4)
}

(1) Universidad Estadual Paulista Júlio de Mesquita Filho - UNESP, Departamento de Ciencia de Información, Av. Hygino Muzzi Filho, 737, Campus Universitário, CEP. 17525900 - Marília, SP - Brasil, Caixa-Postal: 421, milena.rubi@gmail.com. (2) fujita@marilia.unesp.br (3) Universidad Federal de São Carlos - UFSCar, Departamento de Ciencia de Información, Rodovia Washington Luiz, Km 235, Campus Universitário, CEP. 13565905 - São Carlos, SP - Brasil - Caixa-Postal: 676, vboccato@power.ufscar.br.

(4) mariacarolunesp@yahoo.com.br

\begin{abstract}
Resumen
Presenta un estudio diagnóstico con la finalidad de contribuir con apoyos para la elaboración de política de tratamiento de la información documentaria de bibliotecas universitarias. Los resultados demuestran que: los catalogadores siguen una metodología sistematizada y consolidada para la catalogación de forma, pero no demuestran metodología para la catalogación de asunto; la recuperación de la información presenta fallas en lo que respecta a la búsqueda por tema; el lenguaje documentario no atiende de manera satisfactoria a la demanda.
\end{abstract}

Palabras clave: Política del tratamiento de la información documentaria. Catalogación de asunto. Bibliotecas universitarias. Catalogador.

\section{Introducción}

La biblioteca universitaria es un sistema de información que es parte de un sistema de información académico, en el cual la generación de conocimientos es el objetivo de la vida universitaria. Ese contexto nos revela el cambio de paradigmas de la información que, innegablemente, certifica como uno de los más importantes determinantes para la existencia de innovación en lo que se refiere a:

- la forma: la diversidad de formatos exige tratamiento temático y descriptivo compatibles con consecuente modificación de normas, directrices, manuales y metodologías; la co-

\begin{abstract}
This study is aimed at analyzing and describing the cataloguing and indexing procedures in order to obtain the necessary subsides to the working up of a policy of documentary treatment. The partial results show that the cataloguers follow a systematized and consolidated methodology for descriptive cataloguing, but don't show methodology for subject cataloguing; the information retrieval presents failures relating to the search by subject; and the documentary language does not answer adequately the demand, due to the incompatibility between the terms used in the subject cataloguing and those used by the users.
\end{abstract}

Keywords: Documentary information treatment policy. Subject cataloguing. University libraries. Cataloguer.

existencia del formato impreso y del formato electrónico: evolución semejante a la ocurrida con el documento manuscrito para el documento impreso, en ritmo acelerado;

- el acesso: la evolución de la comunicación de datos facilitó el acceso simultáneo de todos a todos los registros;

- el valor: la información registrada, tratada y diseminada por medio del aparato tecnológico de comunicaciones tiene un valor más alto.

La automación de las bibliotecas permitió que los catálogos, antiguamente locales y restrictos a la determinada comunidad, en la actualidad se 
tornen disponibles a través del Internet, atravesando fronteras físicas y temporales.

En nuestro punto de vista, esa visibilidad del catálogo permite que el bibliotecario asuma una nueva responsabilidad comprometida con la construcción de catálogos en bibliotecas universitarias que estén de acuerdo con la realidad no solamente de su comunidad usuaria local, sino como también de una comunidad usuaria potencial virtual, todas cada vez más exigentes.

De la misma manera, es importante afirmar que esa visibilidad del catálogo y la responsabilidad del bibliotecario interpone todo el proceso de catalogación de forma y asunto, tornándose necesario un cambio de postura del bibliotecario, tomando consciencia sobre la importancia de adopción de criterios de calidad para ese proceso que resultará en la recuperación de la información, siendo la política de tratamiento de la información documentaria una de ellas.

La organización de la información en la gestión de bibliotecas universitarias gana una nueva dimensión por el ambiente digital y por la posibilidad de divulgación del conocimiento producido en la universidad.

De esa forma, con la finalidad de contribuir con apoyos para la elaboración de una política de tratamiento de la información documentaria de bibliotecas universitarias, este trabajo presenta un estudio diagnóstico compuesto de tres partes: funcionamiento del tratamiento de informaciones documentales en la perspectiva de la gerencia del sistema de bibliotecas universitarias; procedimientos del tratamiento de informaciones documentales en la perspectiva del catalogador; y descripción de la metodología de colecta de datos para el diagnóstico de tratamiento del contenido de la información documentaria en las bibliotecas universitarias.

\section{Política de tratamiento de la información documentaria en bibliotecas universitarias}

A pesar que la literatura solamente trae elementos que se refieren al respecto específicamente de la política de indexación, consideramos oportuno incluir nuestro estudio para política de tratamiento de información documentaria.

Esto se debe al hecho de que en la Organización de la Información, el tratamiento de la información comporta dos niveles:

- el de la forma: análisis descriptivo o bibliográfico, el tratamiento físico de la información vinculado con el soporte;
- el del contenido: tratamiento temático de la información que se destina a la representación condensada del asunto intrínseco o extrínseco tratado en un determinado documento.

Además, consideramos que no se deben desasociar los dos niveles de tratamiento, toda vez que la biblioteca es compuesta por partes interconectadas (inserción de documentos, clasificación, catalogación, indexación, etc.) con el objetivo común de tener disponible la información de la mejor manera posible.

Se aprecia, por lo tanto, que la indexación y, por consiguiente, su política, es una de las partes de estos sistemas y, como tal, debe ser parte también de la planificación global de los sistemas de información como un parámetro de su administración en el contexto gerencial. De acuerdo con Carneiro (1985, p. 221) una política de indexación

[...] debe servir como una guía para tomar decisiones, debe tener en cuenta los siguientes factores: características y objetivos de la organización, determinantes del tipo de servicio a ser ofrecido; identificación de los usuarios, para la atención de sus necesidades de información y recursos humanos, materiales y financieros, que delimitan el funcionamiento de un sistema de recuperación de informaciones.

Además, la política de tratamiento de información documentaria no debe ser vista como una lista de procedimientos a ser seguidos, sino como una filosofía que discurra los intereses y objetivos de la biblioteca. Esto nos lleva a pensar sobre la indexación desde el punto de vista gerencial y estratégico en el contexto de la biblioteca, otda vez que esta sucede en la entrada y en la salida de informaciones del sistema, confirmándose como la parte más importante porque condiciona los resultados de una estrategia de búsqueda, produciendo una correspondencia necesaria con el asunto investigado en índice (Fujita, 1999).

Esta política se torna importante porque visa la gestión de la información registrada de cara a dar visibilidad en la recuperación de la información para las bibliotecas universitarias, además de identificar conductas teóricas y prácticas de los equipos de tratamiento de la información documentaria involucradas para definir un patrón de cultura organizacional coherente con la demanda de la comunidad académica interna y externa. 


\section{El estudio diagnóstico del contexto en la perspectiva del catalogador y del usuario: procedimientos de aplicación del Protocolo Verbal}

Como metodología, realizamos un estudio diagnóstico de funcionamiento y procedimientos utilizando como estudio de caso la Red de Bibliotecas de la Universidad Estadual Paulista (UNESP).

La Universidad Estadual Paulista "Julio de Mesquita Filho" es una universidad pública, de enseñanza gratuita y está presente en prácticamente todo el territorio paulista. Insertada en este contexto, se encuentra la Coordinación General de Bibliotecas (CGB), responsable por el funcionamiento sistémico de la Red de Bibliotecas de la UNESP, que elaboró el Programa de Gestión 2005-2008, cuya meta es la optimización del sistema de recuperación de informaciones en el Banco de Registros Bibliográficos Athena. Por tanto, necesita poner en práctica las siguientes acciones:

- Elaboración de Política de indexación de la red de Bibliotecas de la UNESP.

- Evaluación del lenguaje documentario utilizado por la red de bibliotecas de la UNESP.

- Poner a disposición, junto a la interfase de búsqueda del banco Athena, una base de autoridades de Bibliodata.

Nuestro estudio se inserta en la primera acción -elaboración de política de indexación de asuntos de la red de Bibliotecas de la UNESP_y pretende describir y analizar los procedimientos de catalogación e indexación, con el propósito de levantar los apoyos necesarios para elaboración de una política en sistema de bibliotecas universitarias.

El estudio diagnóstico está compuesto de tres partes: a) funcionamiento y procedimiento del tratamiento de la información en la Red de Bibliotecas desde la perspectiva de la gerencia del sistema Coordinación General de Bibliotecas; b) funcionamiento y procedimientos de tratamiento de la información en la Red de Bibliotecas desde la perspectiva del catalogador; c) descripción de la metodología de colecta de datos para el diagnóstico del tratamiento de la información en las bibliotecas.

Se utilizaron, para las finalidades de esta investigación, los datos colectados con (Protocolo Verbal Individual con Catalogadores - PVIC) y con alumnos de graduación durante la tarea de recuperación de la información científica (Protocolo verbal Individual con Usuarios - PVIU), además del Protocolo Verbal en Grupos (PVG) aplicado con catalogadores, dirigentes, bibliotecarios de referencia y usuarios investigadores, líderes de grupos de investigación, y alumnos de graduación, así como el desarrollo del cuestionario de diagnóstico organizacional de las bibliotecas (Apéndice).

La metodología de colecta de datos introspectivos consiste en la grabación de la exteriorización verbal de pensamiento durante la realización de una tarea. El "Pensar alto" del sujeto es grabado y copiado literalmente, produciendo protocolos verbales. El lector puede exteriorizar sus procesos mentales en cuanto la información procesada está sobre el foco de su atención. Él lee e interpreta al mismo tiempo, exteriorizando en voz alta todo lo que "pasa por su cabeza" durante la lectura.

Fujita, Nardi y Fagundes (2003) consideran que la técnica introspectiva de "Pensar Alto", o Protocolo Verbal, revela la introspección del lector de forma natural, con ventajas sobre otros tipos de técnicas tales como diarios, cuestionarios o entrevistas porque es la única que ofrece acceso directo al proceso mental de la lectura en cuanto está siendo realizado por el lector, diferente de las otras que revelan solamente una reflexión después del proceso de lectura. De esa forma, la técnica de "Pensar alto" es la única técnica propiamente introspectiva mientras que las otras son de naturaleza retrospectiva.

Cabe destacar que, en términos de Brasil, son pioneros los estudios coordinados por Fujita en lo que respecta a "[...] una observación del proceso de lectura documentaria utilizando una metodología introspectiva del Protocolo Verbal y obtención de relato verbal del proceso mental de lectura y análisis de textos para fines de indexación" (Fujita, 1999, 2003; Fujita, Nardi, Fagundes, 2003).

El desenvolvimiento de las investigaciones en lectura documentaria (Fujita; Cervantes, 2005) ha utilizado modalidades diferentes de Protocolo Verbal. El más utilizado es el Protocolo Verbal Individual en la cual el sujeto es solicitado a "Pensar Alto" y el investigador solamente lo acompaña sin hacer ninguna intervención o comentario. El estudio de Nardi (1999) adaptó el Protocolo Verbal para la investigación con grupos de personas involucrando eventos de lectura realizada para colaborar en la observación de la cognición socialmente construida, denominándolo de Protocolo en grupo.

El protocolo verbal en grupo fue utilizado, en la disertación de Rubi (2004) para analizar el contexto del indexador e investigar su conocimiento sobre política de indexación. Los resultados 
obtenidos demostraron que la metodología puede ser utilizada por sistemas de información para que se tenga acceso al conocimiento del indexador y, de esta manera, propiciar la generación de conocimiento organizacional del sistema.

Para la colecta de datos utilizando la metodología del protocolo verbal, en cualquiera de sus modalidades, es necesario observar los procedimientos que deben ser adoptados en tres momentos distintos: anteriores, durante y posteriores a la colecta de datos. Para la identificación de las diferencias de procedimientos entre las modalidades adoptaremos las siglas PVIC (Protocolo Verbal Individual con Catalogadores), PVIU (Protocolo Verbal Individual con Usuarios) y PVG (Protocolo Verbal en Grupo) después de cada procedimiento usual de las modalidades.

\section{A) Procedimientos anteriores a la colecta de datos}

\section{Definición del universo de la investigación}

PVG: Por intermedio de la Coordinación General de Bibliotecas, se solicitó a las bibliotecas seleccionadas que establezcan una fecha para una reunión, conforme disponibilidad del calendario, con los cinco componentes definidos para la tarea: bibliotecario catalogador, director de la biblioteca, bibliotecario de referencia, docente líder del grupo de investigación, y alumno de graduación. Las reuniones fueron realizadas en las dependencias de las bibliotecas de las unidades seleccionadas, es decir, en el local de trabajo de los participantes.

PVIC: población de bibliotecarios catalogadores representantes de las tres áreas de conocimiento -Humanas, Exactas y Biológicas- respectivamente, Letras, Matemática y Odontología en nueve bibliotecas de la UNESP.

PVIU: muestra de discentes representativos de las tres áreas del conocimiento -Humanas, Exactas y Biológicas - respectivamente, Pedagogía, Ingeniería Civil y Odontología en nueve bibliotecas de la UNESP.

\section{Selección del Texto-Base}

PVG: el texto-base utilizado para la colecta de datos fue seleccionado por tratar de los objetivos de las investigaciones en curso. Para evitar una lectura extensa, fue seleccionado para lectura y discusión en el Protocolo Verbal en Grupo el trecho entre las paginas 205 y 208 por contener los principales aspectos del problema de la investigación del artículo pertenecientes a la discusión que llevaría a relucir las principales reflexiones y percepciones de los integrantes del grupo acerca del contexto de tratamiento documentario de las bibliotecas.

\section{Referencia:}

DIAS, Eduardo Wense; NAVES, Madalena Martins Lopes; MOURA, Maria Aparecida. O usuário-pesquisador e a análise de assunto. Perspect. cienc. inf., Belo Horizonte, v. 6, n. 2, p. 205-221, jul./dez. 2001.

PVIC: Tesis, disertación o libro para catálogo original ( $\mathrm{CO})$; importación de un registro idéntico - identidad total (IT); importación de un registro aprovechable (RA).

PVIU: el usuario ejecutó la búsqueda sin uso del texto-base.

\section{Definición de la tarea:}

PVG: Los tópicos abordados durante las reuniones se refieren al respecto de los itens incluidos en el guión para aplicación del Protocolo Verbal en Grupo. El guión fue definido de acuerdo con los focos de las investigaciones en curso.
Política de indexación y catalogación
Catalogación automatizada x manual
Catalogación de asunto $x$ indexación
Sistemática/metodología para cataloga-
ción/indexación/catalogación de asunto
Manual de indexación
Política de indexación
Interacción entre referencia y proceso técnico
El uso de lenguajes documentarios
Actualización del lenguaje
Adaptación del lenguaje para la comunidad usuaria
El papel del bibliotecario en la construc-
ción/manutención del lenguaje
Pertinencia de este lenguaje con el lenguaje de la comunidad usuaria

\section{Usuarios}
La participación del usuario en la biblioteca
Comportamiento del usuario frente a proble-
mas/dificultades de recuperación de información en la biblioteca
El usuario integrante de comisiones de bibliotecas
Reflexión sobre la acción de la catalogación en la formación
La formación del catalogador (biblioteca-
rio/técnico/auxiliar)
Actualización en servicio
La reflexión sobre la acción de la formación en servicio (cómo sucede, es personal, parte de la jefatura, quién siente la necesidad)
Cursos realizados específicos del área para el ca- talogador.

PVIC: tesis, disertación o libro para catalogación original (CO); importación de un registro idéntico 
- identidad total (IT); importación de un registro aprovechable (RA).

PVIU: investigaciones en el Banco de datos Bibliográficos de la UNESP - ATHENA, por el campo de asunto, formulario de "Investigación Asistida" utilizándose el lenguaje documentario de Bibliodata como agente interruptor para la recuperación de la información.

\section{Selección de los Sujetos:}

PVG: jefe de biblioteca; bibliotecario catalogador, bibliotecario de referencia, usuario docente líder del grupo de investigación y usuario discente.

PVIC: Bibliotecario Catalogador.

PVIU: alumnos de graduación de la primera y última serie de los grupos de Pedagogía, Ingeniería Civil y Odontología en nueve bibliotecas de la UNESP.

\section{Conversación informal con los sujetos:}

PVG, PVIC y PVIU: En esta conversación, el investigador entró en contacto con los sujetos explicando los objetivos de la investigación, la metodología utilizada e indicando el día para la colecta de los datos.

Familiarización con la tarea del "Think Aloud" ("Pensar Alto"):

PVG: esta etapa no ocurre en el protocolo verbal en grupo.

PVIC, PVIU: Esta etapa es muy importante, pues funciona como un entrenamiento para que el sujeto consiga ejecutar la tarea exteriorizando sus pensamientos durante la acción. Como auxilio en esta etapa se utiliza un texto de familiarización (ANEXO).

\section{B) Procedimientos durante la colecta de datos}

Grabación del "Pensar Alto" durante la realización de la tarea (solamente para PVIC y PVIU)

Para toda la exteriorización del pensamiento realizada por el sujeto durante la ejecución de la tarea solicitada fue grabada con el auxilio de un aparato de MP3.

Grabación de la discusión del texto por el grupo de sujetos (solamente para PVG)

El texto-base seleccionado no era de conocimiento de los participantes y se entregó solamente en el momento de la reunión para la colecta de datos. Después de la entrega del textobase, fue solicitada a los participantes una lectura silenciosa y posteriormente se inició una dis- cusión. Toda la discusión del grupo de sujetos participantes fue grabada integralmente por medio de un aparato de MP3 controlado por la investigadora.

\section{Entrevista retrospectiva (optativa):}

PVG: No es muy utilizada en el protocolo verbal en grupo, por eso si es necesaria, debe ser realizada inmediatamente después de terminada la reunión.

PVIC y PVIU: Es un momento en que el investigador tiene la oportunidad de solicitar mayores esclarecimientos sobre algo que no quedó claro, o sobre lo que el sujeto pensó en determinado momento en el que no exteriorizó su pensamiento, permaneciendo callado. La entrevista, si es necesaria, debe ser realizada inmediatamente después de la realización de la tarea.

C) Procedimientos posteriores a la colecta de datos

Transcripción de las grabaciones:

PVG: Después de grabar la discusión del texto por los sujetos, fue realizada la transcripción literal con la identificación de las fuentes de los discursos individuales. Esa identificación fue ficticia para preservar el secreto sobre la identidad de los participantes. Las categorías fueron construidas de acuerdo con los ítems de la guía elaborada para la aplicación del Protocolo Verbal en Grupo. Se resalta que no todas las categorías aparecieron en todas las colectas de datos transcritas.

PVIC y PVIU: Después de la grabación del "Pensar alto" durante la realización de la tarea, fue realizada la transcripción literal de las grabaciones de los discursos de los sujetos.

\section{Resultados}

Los resultados serán presentados teniendo en cuenta las partes que componen el estudio diagnóstico:

- funcionamiento del tratamiento de las informaciones documentales desde la perspectiva de la gerencia del sistema de bibliotecas universitarias - Coordinación General de Bibliotecas (CGB);

- procedimientos del tratamiento de informaciones documentales desde la perspectiva del catalogador;

- descripción de la metodología de colecta de datos para el diagnóstico de tratamiento del 
contenido de la información documentaria en las bibliotecas universitarias.

La Red de Bibliotecas de la UNESP inició su proceso de automación alrededor de 1993/1994 con la participación en la Red BIBLIODATA mantenida por la Fundação Getúlio Vargas (FGV).

Considerándose que la Red de Bibliotecas de la UNESP contaba en la época con un acervo de libros de aproximadamente 800.000 volúmenes, la CGB realizó un estudio comparativo de este acervo con la base de datos de la Red BIBLIODATA, por muestreo, donde se constató una posibilidad de conservación de cerca de $50 \%$ del acervo.

La UNESP decidió iniciar la formación de su Banco de Datos Bibliográficos ATHENA utilizando el proceso de Conversión Retrospectiva RECON (Retrospective Conversion), un modelo ampliamente conocido por Bibliotecas del exterior, para incrementar bases de datos tornando los catálogos on-line más efectivos.

Los acervos solamente podrán ser recuperados con calidad, seguridad y rapidez si el registro, al ser insertado, obedece a criterios rígidos en lo que se refiere tanto a datos descriptivos cuanto a puntos de acceso. La creación de un registro bibliográfico en medio magnético exige la utilización de herramientas como: un código de catalogación y un formato de entrada.

De esta manera, la CGB optó por la utilización del Anglo American Cataloging Rules, $2^{\text {nd }}$ edition $\left(\mathrm{AACR}^{2}\right.$ ) y el formato Machine Readable Cataloging (MARC) desarrollado por la US Library of Congress (LC) - Biblioteca del Congreso Norte-Americano y utilizado internacionalmente, permitiendo a sus usuarios la importación y exportación de registros. El $\mathrm{AACR}^{2}$ es esencial para la perfecta entrada de datos bibliográficos en el formato MARC.

La investigación es iniciada en ATHENA-UEP01 - Banco de datos bibliográficos de la UNESP para verificar si el registro ya se encuentra en la base. En caso positivo, son anotados datos de identificación del registro. En caso negativo, la búsqueda de los registros es realizada en la base de datos cooperativos de la Red BIBLIODATA y los no localizados en esta base serán investigados en la LC.

Existe una clasificación de los registros catalográficos en cuanto a la recuperación: identidad total (IT) cuando los campos, anteriormente descritos, son idénticos tanto en el registro de la base servidora como en lo que se tiene en manos. El registro es grabado en un archivo y será adecuado al Padrón UNESP antes de su impor- tación para la base; registro aprovechable (RA) cuando el registro tiene algunos campos idénticos, pero presenta campos diferentes como, por ejemplo, edición o fecha, este será un registro aprovechable, serán aprovechados los campos iguales y los restantes serán alterados y estos registros serán importados para la base como registros nuevos y posteriormente alimentarán la Red BIBLIODATA; y los registros no localizados (NL) que no fueron encontrados en ninguna de las bases servidoras, para los será realizada la catalogación original.

Las obras no localizadas en BIBLIODATA son investigadas en base LC, cerrando todo el flujo de la investigación. Para las obras no localizadas en esas bases servidoras será realizada la catalogación original.

Los resultados obtenidos del análisis de los datos colectados con la aplicación de la metodología de los protocolos verbales en grupo e individual demuestran que los catalogadores siguen una metodología sistematizada y consolidada por la literatura para la catalogación de forma (tratamiento físico), pero no demuestran metodología para la catalogación de asunto (tratamiento temático). Por otro lado, los resultados del análisis de los datos colectados con el protocolo verbal en grupo, que permite observar la visión de los usuarios en relación a los catálogos, demostraron que la recuperación de la información presenta fallas en lo que se refiere a la investigación por asunto, confirmando los resultados del protocolo verbal individual.

Así mismo, quedó demostrado que el lenguaje documentario no atiende de manera satisfactoria a la demanda, no existiendo compatibilidad entre los términos utilizados en la catalogación de asunto y aquéllos utilizados en las investigaciones por los usuarios. Consideramos que esa situación ocurre debido a tres factores: falta de discernimiento por parte del bibliotecario sobre el proceso de indexación que él realiza durante la catalogación; falta de política de tratamiento de la información documentaria consolidada; y lenguaje documentario que no se ajusta al lenguaje del usuario.

\section{Consideraciones finales}

El conocimiento construido en investigación es difundido y ampliado en la enseñanza ( $\mathrm{y}$ viceversa) y socializado en la extensión, contexto del que nuevamente recibiremos apoyos que impliquen la creación de nuevos conocimientos. Desde esta perspectiva, la universidad actúa como organismo generador, transmisor y receptor de conocimientos y la biblioteca universitaria se torna consciente de su función intermediaria 
realizando los procesos documentarios y preservando la información para su próxima transformación en conocimiento en un espiral de evolución científica y tecnológica.

La evolución de los soportes de información y los cambios de paradigma dentro del contexto de la biblioteca universitaria deben hacer que el bibliotecario asuma una nueva posición, en cuanto constructor de formas de representación del conocimiento, toda vez que su función es justamente hacer puentes entre los documentos almacenados y las preguntas de búsqueda de los usuarios.

En este contexto, se torna primordial la adopción de criterios y prácticas, que se ajusten al nuevo escenario, y al acceso de la información por medio de los catálogos on-line y la atención a los usuarios remotos. Esto constituye un desafío para el profesional, ya que demanda mayor compromiso intelectual y requiere cambios de postura en la conducta profesional.

De esta manera, la política de tratamiento de información documentaria y todo aquello que la compone -metodologías para representación descriptiva y temática, lenguajes documentarios, estudio de usuario, entre otros- necesita de mayor atención en lo que respecta a la elaboración de normas, procedimientos y técnicas, su implantación, implementación y evaluación para que la función de las bibliotecas universitarias sea mantenida y actualizada de acuerdo con los cambios de la sociedad.

Se recomienda, por consiguiente, la elaboración de políticas de tratamiento de informaciones documentarias para bibliotecas universitarias que contemplen tanto la cuestión del tratamiento de forma cuanto la de contenido y que reflejen una nueva filosofía de trabajo del bibliotecario.

\section{Notas}

(1) Milena Polsinelli Rubi: Alumna de Doctorado del Programa de Post-Grado en Ciencia de Información de la Facultad de Filosofía y Ciencias de la Universidad Estadual Paulista - UNESP, Campus de Marília - Brasil, con beca otorgada por CAPES.

(2) Mariângela Spotti Lopes Fujita: Profesora Adjunta del Departamento de Ciencia de Información de la Facultad de Filosofía y Ciencias de la Universidad Estadual Paulista - UNESP, Campus de Marília Brasil.

(3) Vera Regina Casari Boccato: Profesora Asistente del Departamento de Ciencia de Información de la Universidad Federal de São Carlos-UFSCar-Brasil y alumna de Doctorado del Programa de PostGrado en Ciencia de Información de la Facultad de Filosofía y Ciencias de la Universidad Estadual Paulista - UNESP, Campus de Marília - Brasil.
(4) Maria Carolina Gonçalves: Alumna de Maestría del Programa de Post-Grado en Ciencia de Información de la Facultad de Filosofía y Ciencias de la Universidad Estadual Paulista - UNESP, Campus de Marília - Brasil, con beca otorgada por FAPESP.

\section{Referencias}

AMEIDA, M. C. B. de. (2005). Diagnóstico organizacional. // AMEIDA, M. C. B. de. Planejamento de bibliotecas e serviços de informação. 2. ed. rev.e ampl. Brasília: Briquet de Lemos, 2005. p. 53-92.

Carneiro, M. V. (1985). Diretrizes para uma política de indexação. // Revista da Escola de Biblioteconomia da ufmg. 14:2 (Set. 1985) 221-241.

Fujita, M. S. L. (1999). A leitura do indexador: estudo de observação. // Perspectivas em Ciência da Informação. 4:1 (1999) 101-116.

Fujita, M. S. L. (2003). A leitura documentária do indexador: aspectos cognitivos e lingüísticos influentes na formação do leitor profissional. Marília: Faculdade de Filosofia e Ciências, Universidade Estadual Paulista, 2003. Tese de livre-docência.

Fujita, M. S. L.; Cervantes, B. M. N. (2005). Abordagem Cognitiva do Protocolo Verbal na Confirmação de Termos para a Construção de Linguagem Documentária em Inteligência Competitiva. // Valentin, M. L. P. (org.). Métodos qualitativos de pesquisa em Ciência da Informação. São Paulo: Polis, 2005. 1:29-57.

Fujita, M. S. L..; Nardi, M.I.A.; Fagundes, S.A. (2003). Observing documentary reading by verbal protocol. // Information Research. 8:4 (2003). http://informationr. net/ir/8-4/paper155.html. (2006-06-10).

Nardi, M. I. A. (1999). A metáfora e a prática de leitura como evento social: instrumentos do pensar a biblioteconomia do futuro. São Paulo: Pontifícia Universidade Católica de São Paulo, 1999. Tese de Doutorado.

Rubi, M. P. (2004). A política de indexação na perspectiva do conhecimento organizacional. 2004. Marília: Faculdade de Filosofia e Ciências, Universidade Estadual Paulista, 2004. Dissertação de Mestrado. 


\section{Anexo I}

Proyecto Política de Tratamiento de Información Documentaria de la Red de Bibliotecas de la UNESP: diagnóstico organizacional

Está en desarrollo el proyecto de investigación intitulado "Política de tratamiento de información documentaria de la Red de Bibliotecas de la UNESP" bajo la coordinación de la Prof. Dra. Mariângela Spotti Lopes Fujita, con un equipo formado por bibliotecarias de la Coordinación General de Bibliotecas y alumnas de post-grado en Ciencias de Información (Milena Polsinelli Rubi; Vera Regina Casari Boccato y Maria Carolina Gonçalves) con financiamiento del Fondo de Investigación de la Facultad de Filosofía y Ciencias, UNESP, Campus de Marília, con el objetivo de elaborar una política de tratamiento de informaciones de la Red de Bibliotecas UNESP. En este sentido, se torna necesario la realización de un estudio diagnóstico de funcionamiento y procedimientos del tratamiento de información documentaria de su Biblioteca, motivo por el cual solicitamos la colaboración de la Dirección de esa Biblioteca en el sentido de responder el cuestionario, anexado en la parte inferior, juntamente con el catalogador participante de esta investigación.

\section{Diagnóstico organizacional}

1) Nombre oficial de la Biblioteca:

2) Espacio físico: especificar un área física en $m^{2}$

3) Cuál(es) es(son) el(las) área(s) de actuación/especialidad(es) de la biblioteca

4) Estructura organizacional de la biblioteca

a) posee organograma? SI ( ) NO ( )

b) la biblioteca está próxima a la alta administración (al poder decisorio de la institución perteneciente)?

SI ( ) NO ( ). Cuál?

5) Administración de la Biblioteca

a)centralizada ( )

b)participativa ( )

6) La biblioteca realiza una planificación anual de sus actividades?

$\mathrm{SI}$ ( ) NO ( ).

Si la respuesta es $\mathrm{SI}$, ejemplifique una actividad descrita en el plano anual.

7) El usuario participa en la planificación de las actividades de la biblioteca?

$\mathrm{SI}$ ( ) NO ( )

8) El usuario participa en la comisión de biblioteca?

$\mathrm{SI}$ ( ) NO ( )

9) Cuál es el flujo de trabajo de los servicios de procesamiento técnico y de referencia?

10) Proyectos

a) Cuáles son los tipos de proyectos que la biblioteca desarrolla?

b) En ese proceso, es involucrado:

i. un equipo de la biblioteca ( )

ii. otras instituciones/unidades de información ( ) iii. un equipo de la biblioteca y también otras instituciones/unidades de información ( )

\section{1) Documentación técnica}

a) Posee manuales de servicios? SI ( ) NO ( )

b) Cuáles?

12) Documentación administrativa: Posee?

a) Regimiento de la Biblioteca: SI ( ) NO ( )

b) Reglamento de servicios: SI ( ) NO ( ). Si la respuesta es SI, cuáles?

c) Comisión de biblioteca: SI ( ) NO ( )

d) Reglamento de la comisión de biblioteca: SI ( ) NO ( )

\section{3) Recursos Humanos}

a) Cuantificar:

- bibliotecarios (Total):

- número de bibliotecarios que desenvuelven la actividad de referencia (atención al usuario):

- número de bibliotecarios que desarrollan la actividad de catalogación en el Banco de Datos Bibliográficos Athena:

- auxiliares de biblioteca:

- auxiliares de servicios generales:

- técnico en Informática:

b) posee programa de capacitación para los recursos humanos de la biblioteca?

SI ( ) NO ( ). Si la respuesta es SI, cuáles?

14) Cuáles competencias son importantes para su función en la biblioteca?

15) Cuáles son las principales necesidades percibidas por usted en relación a su formación en servicio?

16) Usted considera importante reflexionar sobre su actuación profesional durante la propia acción?

SI ( ) NO ( ). Por qué? 


\section{7) Acervo}

Tipos de materiales (Cuantificarlos):

OBS: en caso de periódicos, cuantificar el número de títulos y de volúmenes.

1 Procesamiento técnico: catalogación:

(1) sigue padrones: SI ( ) NO ( )

(2) cuáles (códigos de catalogación, manuales)?

18) Procesamiento técnico: catalogación de asunto

a) Cuál es el vocabulario controlado/lenguaje documentario (nombre) utilizada por el Sistema Bibliodata para la realización de la actividad de catalogación de asunto y para la realización de búsquedas en el Banco Athena?

b) El vocabulario controlado/lenguaje documentario utilizado por el Sistema Bibliodata para la realización de la actividad de catalogación de asunto atiende satisfactoriamente las necesidades de la realización de la actividad catalogación de asunto?

\section{9) Informatización}

Posee servicios/productos automatizados?

SI ( ) NO ( ). Si la respuesta es SI, especificar:

\section{0) Recuperación de la información}

a) el Banco de datos Bibliográficos de la UNESP Athena es muy utilizado por los usuarios?

b) específicamente, cuál(es) es(son) el(los) campo(s) de recuperación de la información más utilizado(s)?

( ) Todos los Campos ( ) Autor ( ) Título ( ) Asunto

\section{1) Usuarios}

a) Cuantificar el número de usuarios:

reales

potenciales

b) Cuál(es) es (son) la (las) categoría(s) de usuarios que la biblioteca posee:

Graduación ( )

Post-grado:

( ) Especialización ( ) Maestría ( ) Doctorado ( ) Docente

( ) Todas las categorías anteriormente citadas

c) Cuantificar y caracterizar los grupos de investigaciones existentes en la Unidad.

d) Existe algún servicio de la biblioteca que prioriza los grupos de investigaciones de su Unidad?

SI ( ) NO ( ). Si la respuesta es SI, cuáles?

e) Cómo la biblioteca prioriza los grupos de investigación de la Unidad en el desenvolvimiento de sus servicios?

\section{2) Entrenamientos de usuarios}

a) ofrecido regularmente:

SI ( ) NO ( )

b) direccionado especificamente a una determinada categoría: SI ( ) NO ( ). Si la respuesta es SI, cuál?

c) es ofrecido un entrenamiento específico para la utilización del Banco de Datos Bibliográficos de la UNESP - Athena? SI ( ) NO ( )

d)la búsqueda por el Campo de Asunto es presentada/enseñada a los usuarios?

SI ( ) NO ( )

e)la recuperación de la información por el campo de asunto realizada por el usuario en el Banco Athena atiende satisfactoriamente a sus necesidades de búsqueda?

SI ( ) NO ( ). Si la respuesta es NO, por qué?

\section{3) Comunicación}

a )Cómo es realizada la divulgación de los servicios/productos realizados/disponibles por la biblioteca a los usuarios?

b) la biblioteca poseei:

( ) boletines eletrónicos

( ) boletines impresos

() Ambos

( ) Otros materiales de divulgación. Cuál(es)?

c) cómo es realizada la comunicación entre los funcionarios de la biblioteca:
( ) por e-mail
() teléfono
( ) memorandos
() personalmente
( ) otros medios. Cuál(es)?

\section{4) Relación con instituciones afines}

a) cómo es la relación de la biblioteca con las bibliotecas de la(s) misma(s) área(s) de actuación integrantes del Sistema de Bibliotecas de la UNESP?

( ) Muy frecuente ( ) Frecuente ( ) Temporal ( ) No Existe

Especifique cuáles son esas bibliotecas

b) son realizadas reuniones de trabajo internas y/o conjuntas con esas bibliotecas?

$\mathrm{SI}($ ) NO ( )

\section{5) Evaluación}

a) es realizada regularmente? $\mathrm{SI}$ ( ) NO ( )

b) abarca todos los servicios y productos realizados/disponibles por la biblioteca?

SI ( ) NO ( ). Si la respuesta es SI, cuáles? 


\section{Anexo II}

\section{Instrucciones a los informantes sobre la técnica del "pensar alto" o protocolo verbal}

Lo que vamos a realizar ahora es una actividad de familiarización con la técnica de colecta de datos que será utilizada en nuestro estudio.

Todo lo que usted tiene que hacer es realizar sus investigaciones de la misma manera con la que usted acostumbra a efectuar sus actividades profesionales.

Durante toda la investigación usted necesita "pensar alto". Intente imaginar a usted solo en un recinto investigando un asunto de su área. En situaciones como esa, ¿no se le ocurrió ya comenzar a hablar espontáneamente en voz alta, exteriorizando sus raciocinios, sus mecanismos mentales para conseguir investigar? En este proceso, el individuo "piensa en voz alta" verbalizando espontánea y casi inconscientemente sus pensamientos, preguntas, sus búsquedas para eventuales problemas de comprensión, su manera singular de extraer significado de una investigación.

Un ejemplo bastante claro de exteriorización del pensamiento durante la realización de una tarea (y que ocurre con la mayoría de las personas) es el "pensar alto" espontáneo durante la realización de un problema matemático.

¿Puede tener una idea de cómo funciona esa técnica? Corresponde a la verbalización de su habla interna, su pensamiento.
Ahora, la tarea que usted va a realizar es una investigación de un asunto en la Base de datos LILACS utilizando el campo de descriptor de asunto que le va a ser presentado... y, por favor, recuerde de que es necesario "pensar alto" durante toda la investigación.

Usted probablemente encontrará pasajes muy claros y fáciles de comprender, otros podrán obligarle a dar una "paradita" para pensar un poco más... Todo depende de su propio estilo.

Recuerde, que en esos momentos de parar para pensar un poco más o resolver algún problema, usted debe intentar exteriorizar todo lo que pasa por su cabeza.

Si en algún momento de la investigación, encuentra difícil hablar y pensar simultáneamente, podrá suministrar una explicación de cómo usted buscó la solución para un problema de comprensión.

En la medida de lo posible, intente realizar esfuerzos para "pensar alto" durante su proceso de investigación. Es un proceso único en que hablar es pensar.

Intente olvidar la presencia de la observadora/investigadora. Ella estará presente para recordarle que es necesario "pensar alto" todo el tiempo. Intente actuar naturalmente cuando sea posible, como si usted estuviera solo.

Aténgase solamente a la tarea que usted debe realizar. 